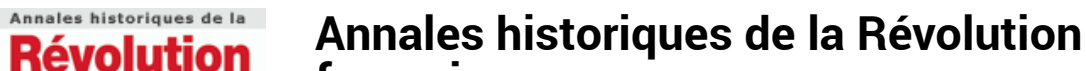

française française

323 | janvier-mars 2001

Varia

\section{Olympes de Gouges. Die Rechte der Frau. 1791}

\section{Marita Gilli}

\section{OpenEdition}

\section{Journals}

Édition électronique

URL : https://journals.openedition.org/ahrf/1046

DOI : 10.4000/ahrf.1046

ISSN : 1952-403X

Éditeur :

Armand Colin, Société des études robespierristes

\section{Édition imprimée}

Date de publication : 1 mars 2001

Pagination : 131

ISSN : 0003-4436

\section{Référence électronique}

Marita Gilli, «Olympes de Gouges. Die Rechte der Frau. 1791 », Annales historiques de la Révolution française [En ligne], 323 | janvier-mars 2001, mis en ligne le 21 avril 2004, consulté le 23 avril 2022 URL : http://journals.openedition.org/ahrf/1046 ; DOI : https://doi.org/10.4000/ahrf.1046

Ce document a été généré automatiquement le 23 avril 2022.

Tous droits réservés 


\title{
Olympes de Gouges. Die Rechte der Frau. 1791
}

\author{
Marita Gilli
}

\section{RÉFÉRENCE}

Karl Heinz Burmeister, Olympes de Gouges. Die Rechte der Frau. 1791, Stämpfli Verlag A G Bern, Manz Wien 1999, 197 p.

1 Ce joli petit livre consacré à Olympes de Gouges comporte une importante introduction qui retrace le contexte dans lequel l'auteur a conçu Les droits de la femme. En effet, la Déclaration des droits de l'homme et du citoyen d'août 1789 ne tenait pas compte des droits des femmes et celles-ci l'avaient interprétée comme valable pour les hommes seulement. Deux ans plus tard, la Déclaration des droits de la femme et de la citoyenne rédigée par Olympe de Gouges se voulait le pendant de la première et elle était la première proclamation féministe d'égalité entre les hommes et les femmes.

2 Dans l'image du monde des Lumières, les femmes et les Noirs étaient considérés comme des «hommes manqués» et ne faisaient pas partie des citoyens potentiels. On aurait pu s'attendre à ce que la Révolution leur apporte l'égalité; comme l'a dit Sophie de Condorcet: «Dans un pays où on leur coupe la tête, il est naturel qu'elles veuillent savoir pourquoi». Or, la Révolution ne leur apporte pas grand chose. Dans son écrit Über die bürgerliche Verbesserung der Weiber, Theodor Gottlieb von Hippel s'étonne que la Révolution n'ait pas amélioré le statut des femmes, alors que celles-ci s'étaient engagées et montaient volontiers à la tribune. Olympe de Gouges qui a revendiqué l'égalité totale des femmes, leur indépendance économique, l'introduction du divorce et de la reconnaissance de paternité a été ensuite oubliée, surtout en Allemagne où on ne trouve son nom que dans les très grandes encyclopédies savantes et il a fallu attendre le bicentenaire de la Révolution pour qu'elle soit connue dans ce pays. Hannelore Schröder lui a consacré d'importantes recherches à partir de 1973. À l'heure actuelle, elle a retrouvé la place qui lui revient dans l'histoire de la littérature française. 
3 L'introduction consacre un développement à la situation de la femme dans les Lumières, à la culture des femmes telle qu'elle était conçue au XvIIIe siècle, à la femme dans la Révolution. Est ensuite présentée une biographie d'Olympe de Gouges, puis une longue introduction à son œuvre Les droits de la femme au centre de laquelle se trouve la Déclaration des droits de la femme et de la citoyenne. Sont ainsi indiquées les dates de parution de l'ouvrage et une longue étude sur les précurseurs éclaire le texte. Puis l'auteur se penche sur les différentes parties de l'ouvage: la dédicace, l'introduction, la déclaration, elle-même divisée en un préambule et 17 articles, le postambule et la Forme du Contrat social de l'Homme et de la Femme qui clôt l'œuvre. Enfin, l'auteur présente une interprétation, estimant que l'œuvre est loin d'être utopique et fait l'étude de sa réception. Il présente ensuite le texte intégral de cette déclaration et sa traduction en allemand que l'on doit à Ulrike Längle.

4 D'un format agréable et pourvu de belles illustrations, l'ouvrage convient aussi bien au néophyte désireux de connaître Olympe de Gouges qu'au chercheur, dans la mesure où il est accompagné de tout un appareil critique: notes, indication des sources, bibliographie. 